\title{
Commentaries
}

\section{Effect of growth hormone and glutamine on the short bowel: five years later}

Short bowel syndrome is a condition marked by weight, fluid, and nutrient loss in the absence of adequate small intestine length. In adults this is usually the result of surgical intestinal resection for Crohn's disease, acute bowel infarction, radiation strictures, and adhesive obstruction. Patients with a residual small bowel length of $100 \mathrm{~cm}$ (normal $600 \mathrm{~cm}$ ) or less usually require parenteral nutrition for survival. Prior to the discovery of total parenteral nutrition (TPN) in 1968 by Dudrick and colleagues, patients with short bowel often died as a result of fluid and nutritional deficiency. ${ }^{12}$ During the past three decades we have learned that parenteral nutrition can be given in the home environment (home parenteral nutrition ( $\mathrm{HPN})$ ) with good results. ${ }^{3}$ However, there are complications associated with long term HPN which include catheter sepsis, liver injury, and bone disease. Daily administration of HPN also requires dedication and planning by patients with regard to sterile catheter technique and travel away from the usual home environment. HPN can be expensive if given over an extended period of time.

Fortunately for some patients, the remaining small intestine undergoes clinically significant structural and functional changes (intestinal adaptation) following surgical resection which may eliminate the need for lifelong TPN. The process of intestinal adaptation can be observed clinically by documentation of weight, fluid, and electrolyte stabilisation. Over a 3-12 month period following resection, patients usually declare their need for lifelong TPN.

Various medical and surgical treatments have been attempted over the past 20 years to increase the surface area of the residual small intestine with the aim of increasing fluid and nutrient absorption. Perhaps the most interesting was the published work of Byrne et al in 1995. ${ }^{4}$ These investigators treated eight patients with short bowel syndrome who had been dependent on TPN for many years, with human recombinant growth hormone, glutamine, and a high carbohydrate/low fat diet for three weeks. They reported a significant increase in total caloric, carbohydrate, protein, and fluid absorption. However, they found no change in fat absorption however. In a subsequent study using the same treatment, where the end point was discontinuation of TPN rather than nutrient or fluid absorption, they were able to wean $40 \%$ of their patients off TPN. ${ }^{5}$ Unfortunately, evaluating only this end point does not directly answer the question of whether the treatment was solely responsible for the positive effects observed as absorptive studies were not performed. It is possible that these patients were not truly "TPN dependent" and the same results could have been found with conventional treatment alone. These two studies have been criticised for not including a placebo arm and making this method of treatment commercial without more objective data.
In this issue of Gut a group of investigators from Copenhagen who have a great deal of experience in short bowel syndrome report their results in eight short bowel patients treated with growth hormone, glutamine, and their usual diet (see page 199). ${ }^{6}$ Unlike the previous studies, this was a randomised placebo controlled trial. They did not find a significant increase in fluid or caloric absorption in those patients with and without colons compared with placebo. Their reasons for not choosing to use a high carbohydrate/ low fat diet given their earlier results are not clear. ${ }^{7}$ The lack of positive findings compared with the study of Byrne et al may be a reflection of diet and not growth hormone and glutamine. The results of Szkudlarek et al are consistent with the work from our institution using the same experimental design. ${ }^{8}$ However, we used a high carbohydrate/low fat diet throughout the study. Although we reported a transient increase in body weight in those treated compared with placebo, we later reported the body composition data in this same group of patients and found that extracellular fluid retention, most likely from growth hormone, was responsible for the effects observed. ${ }^{9}$ Similarly, Szkudlarek et al reported side effects from extracellular fluid retention in all eight patients. One patient required surgery for severe carpal tunnel syndrome.

One observation consistent in this current study as well as in those of Byrne et al and Scolapio et al is that fat absorption was unchanged. I believe this is a good indication that treatment with growth hormone and glutamine has little if any clinical effect on small bowel absorption. Five years and two randomised controlled studies later, I believe the findings reported by Byrne et al are the result of meticulous dietary counselling and not necessarily those of growth hormone and glutamine.

J S SCOLAPIO

Director of Parenteral and Enteral Nutrition,

Division of Gastroenterology and Hepatology,

Mayo Clinic, Facksonville, Florida, USA

Email: scolapio.james@mayo.edu

1 Dudrick SJ, Wilmore DW, Vars HM, Rhoads JE. Long-term total parenteral nutrition with growth development, and positive nitrogen balance. Surgery 1968;64:134-42.

2 Dudrick SJ, Wilmore DW, Vars HM, Rhoads JE. Can intravenous feeding as the sole means of nutrition support growth in the child and restore weight loss in the adult? An affirmative answer. Ann Surg 1969;169:974-84.

3 Scolapio JS, Fleming CR, Kelly DG, et al. Survival of home parenteral nutrition-treated patients: 20 years of experience at the Mayo Clinic. Mayo Clin Proc 1999;74:217-22.

4 Byrne TA, Morrissey TB, Nattakom TV, et al. Growth hormone, glutamine and a modified diet enhance nutrient absorption in patients with severe short bowel syndrome. FPEN f Parenter Enteral Nutr 1995;19:296-302.

5 Byrne TA, Persinger RL, Young LS, et al. A new treatment for patients with short-bowel syndrome. Growth hormone, glutamine and a modified diet. Ann Surg 1995;222:243-54

6 Szkudlarek J, Jeppesen PB, Mortensen PB. Effect of high dose growth hormone with glutamine and no change in diet on intestinal absorption in short bowel patients: a randomised, double blind, crossover, placebo controlled study. Gut 2000; 47:199-205.

7 Nordgaard I, Hansen BS, Mortensen PB. Importance of colonic support for energy absorption as small-bowel failure proceeds. Am $\mathcal{f}$ Clin Nutr 1996;64:222-31.

8 Scolapio JS, Fleming CR, Camilleri MC, et al. Effect of growth hormone, glutamine and diet in short bowel syndrome. A randomized controlled study. Gastroenterology 1997;113:1074-81.

9 Scoalpio JS. Effect of growth hormone, glutamine and diet on body composition in short bowel syndrome. FPEN F Parenter Enteral Nutr 1999;23:30913. 


\section{Screening and shouting about $\mathrm{HCV}$}

In this month's Gut, Ward and colleagues ${ }^{1}$ report their study of the prevalence of hepatitis C virus (HCV) in women attending an inner London obstetric department (see page 277). The study was well designed and carefully executed. The methodology is valid and the findings are important. The authors make three key observations: that the prevalence of $\mathrm{HCV}$ in this population is high $(0.8 \%)$; that uptake and acceptability of screening for HCV is good; and that many of the cases could not be identified by clinical criteria alone. Each of these findings is important in its own right. Together they reaffirm the importance of hepatitis $\mathrm{C}$ as a healthcare problem. They support the case for further investigation of screening strategies for $\mathrm{HCV}$ and suggest that antenatal screening should be considered carefully.

Accurate knowledge of the prevalence of a disease is particularly important when it is serious and treatable. In the 1970 s the disease later recognised as hepatitis C was thought to be an inconsequential transaminitis that followed blood transfusion. Since the cloning of $\mathrm{HCV}$ in 1989, increasing knowledge of the natural history of hepatitis $C$ has revealed a more serious outcome, at least for the minority of individuals who develop progressive fibrosis leading to cirrhosis, liver failure, and liver cancer. ${ }^{2}$ In the past year, effective treatment has been licensed ${ }^{3}$ and even more effective therapies may follow. Now that more is known of the natural history of hepatitis $\mathrm{C}$ and the clinical and cost effectiveness of treatment, accurate knowledge of the true prevalence of $\mathrm{HCV}$ infection is very important for those planning, funding, and delivering care to $\mathrm{HCV}$ infected individuals.

Early studies found that the prevalence of HCV infection was as low as $0.04 \%$ in populations that could be readily accessed, such as blood donors. ${ }^{4}$ However, there are many reasons why this group may not be representative of the wider population, not least of all because they are carefully selected for being in good health. Following recognition of intravenous drug use as a major route of transmission of $\mathrm{HCV}$, studies were conducted to measure the prevalence among injecting drug users; approximately $67 \%$ of subjects tested were infected. ${ }^{5}$ Such widely diverse measurements in specific groups demonstrate the importance of selecting a representative sample to determine the prevalence in the general population. Screening studies in the general population are problematic, particularly when identification of infected individuals carries serious consequences for the patient, the carer, and the healthcare system. Pilot studies in discrete, representative, and informative populations are preferred.

Ward et al report the prevalence of $\mathrm{HCV}$ infection in antenatal clinic attendees in inner London. Antenatal clinic attendees are a particularly interesting sample population with specific demographic characteristics that introduce biases, some of which are desirable and others which are not. The women are generally of an age when they are likely to have been exposed to HCV if at risk. If infected they are likely to be early enough in the course of their disease to benefit from treatment. As a group, clinic attendees are likely to be accepting of a medical intervention that may benefit themselves and their children and of a screening test that can be incorporated within their routine antenatal care. Pregnancy is well distributed through social classes (although rates may differ). The disadvantage of selecting the antenatal population for screening is that HCV infection is known to be less common in women than in men. Also, by selecting only those women who can conceive, the study will exclude women rendered infertile as a result of liver disease. Thus the prevalence of HCV infection measured in this study is likely to be an underestimate of the true prevalence in the general population. The investigators work in inner London and care for an ethnically diverse population. However, this diversity is probably little different from that in most UK cities. Furthermore, Caucasian women were represented at the same frequency in the HCV positive group as in the whole sample, suggesting that ethnicity was not a major determinant of infection.

Ward et al found evidence of $\mathrm{HCV}$ infection in $0.8 \%$ of their sample of which $0.6 \%$ were viraemic. The ratio of viraemic to non-viraemic, antibody positive individuals is in keeping with knowledge of the natural history of HCV infection. These relatively high levels of prevalence are in keeping with other reports of antenatal screening ${ }^{6}$ and anonymous testing of antenatal attendees. ${ }^{7}$ If prevalence in the male population is correspondingly higher, the true population wide prevalence of $\mathrm{HCV}$ is likely to be more than $1 \%$ and similar to that reported in the USA. ${ }^{8}$ Such a high level of infection establishes hepatitis $\mathrm{C}$ as a major healthcare problem for the 21 st century and one that cannot and will not be ignored. These findings have serious consequences for the National Health Service and Department of Health in the UK.

The present study examined more than just prevalence and formed a pilot screening study. The National Screening Committee set stringent criteria (based on those of Wilson and Junger, adapted by Gray) ${ }^{9}$ which must be met before a screening programme can be introduced. These criteria include demonstration of the importance of the health problem, an accepted treatment, an accurate, reproducible and acceptable test, and an adequate window of opportunity when patients with positive tests can be identified and treated. While aspects of testing are widely accepted, some of the other criteria remain controversial. Effective treatment is available but clinicians and those funding healthcare are at odds over the provision of treatment. The judgement of NICE is awaited. The investigators have added to the evidence that $\mathrm{HCV}$ is an important health problem but the wider public may yet require further convincing. The authors have measured the uptake and acceptability of screening in the antenatal clinic and found both to be high. In some ways this is unsurprising for the reasons stated above. A group of women who have already chosen to attend clinic, about to give birth to children in whom the majority will recognise considerable investment, might be assumed to be motivated. Women, unlike men, are already provided with national screening programmes for cervical and breast cancers and so might be more compliant with further screening programmes than men. However, without the evidence contained in this report, larger screening pilots and even national programmes could not be developed. The time has come for a good quality randomised controlled trial of screening for HCV.

One of the most interesting findings of Ward et al's study is that clinical criteria failed to identify those women who had been infected with $\mathrm{HCV}$; both those who remain viraemic as well as those who are merely HCV antibody positive. Early investigations found that infection could not be attributed to a specific risk factor in 
up to $40 \%$ of infected individuals..$^{10}$ Most hepatologists, and particularly nurses who work with them, will have been aware that close questioning in private reveals the majority of unattributable exposures to be related in some way to intravenous drug use. In common with most previous studies, the authors found that exposure to illicit drugs, or a partner with a history of intravenous drug use or $\mathrm{HCV}$ infection, was over represented in the infected population. Unless contextually relevant, few patients are likely to admit to these risk factors when first interviewed. Anyone working in the field of viral hepatitis will be well aware that intravenous drug users come in all shapes and sizes. The stereotypic view of the opiate addict as a pale and spotty waif with pinpoint pupils has many exceptions and is in marked contrast with many occasional users of illicit stimulants such as amphetamine and cocaine who make up the majority of intravenous drug users in the UK. While the former population may be at greater individual risk the latter are more numerous and are likely to be at significant risk of $\mathrm{HCV}$. In the absence of any reliable clinical discriminant test for HCV, a low threshold for the widespread use of a diagnostic test for the virus is indicated. Tattooing and piercing was also over represented among the HCV infected individuals, confirming the importance of these independent risks.
The report by Ward et al carries important messages about HCV. Patients, clinicians, and those planning healthcare alike should heed them. Further research into screening for $\mathrm{HCV}$ is required.

Liver Group, Division of Cell and Molecular Medicine,

W ROSENBERG

University of Southampton,

Mailpoint 811 Level D South Block, Southampton General Hospital,

Tremona Road, Southampton SO16 6YD, UK

Email:wmr@soton.ac.uk

1 Ward C, Tudor-Williams G, Cotzias T, Hargreaves S, Regan L, Foster GR. Prevalence of hepatitis $C$ among pregnant women attending an inner London obstetric department: uptake and acceptability of named antenatal London obstetric department:

Di Bisceglie AM. Hepatitis C. Lancet 1998;351:351-5.

3 Poynard T, Marcellin P, Lee SS, et al. Randomised trial of interferon alpha $2 \mathrm{~b}$ Poynard T, Marcellin P, Lee SS, et al. Randomised trial of interferon alpha $2 \mathrm{~b}$
plus ribavirin for 48 weeks or for 24 weeks versus interferon alpha $2 \mathrm{~b}$ plus placebo for 48 weeks for treatment of chronic infection with hepatitis C virus. International Hepatitis Interventional Therapy Group (IHIT). Lancet 1998;352:1426-32.

4 McLindon JP, Paver WK, Babbs C, et al. Hepatitis C-related chronic liver disease among asymptomatic blood donors in the north west of England. $\mathcal{F}$ Infect 1995;30:253-9

5 Lamden KH, Kennedy N, Beeching NJ, et al. Hepatitis B and hepatitis C virus infections: risk factors among drug users in Northwest England. $\mathcal{f}$ Infect 1998;37:260-9.

6 Garner JJ, Gaughwin M, Dodding J, Wilson K. Prevalence of hepatitis C infection in pregnant women in South Australia. Med f Aust 1997;167:470-2.

7 Puro V, Girardi E, Ippolito G, et al. Prevalence of hepatitis B and C viruses and human immupodeficiency virus infections in women of reproductive age. Br f Obstet Gynaecol 1992;99:598-600.

8 Alter MJ. Hepatitis C virus infection in the United States. F Hepatol 1999;31(suppl 1):88-91.

9 Gray JA. Evidence-based healthcare. London: Churchill Livingstone, 1997.

10 Conry Cantilena C, VanRaden M, Gibble J, et al. Routes of infection, Conry Cantilena C, VanRaden $\mathrm{M}$, Gibble J, et al. Routes of infection,
viremia, and liver disease in blood donors found to have hepatitis $\mathrm{C}$ virus viremia, and liver disease in blood donors
infection. $N$ Engl f Med 1996;334:1691-6.
Hepatorenal syndrome is a serious life threatening complication in end stage liver disease. A recent consensus conference has agreed definitions for hepatorenal syndrome and divided the syndrome into types I and II. ${ }^{1}$ Type I is characterised by rapidly progressive renal failure with a doubling of serum creatinine to a level greater than $2.5 \mathrm{mg} / \mathrm{dl}$ or a halving of creatinine clearance to less than $20 \mathrm{ml} / \mathrm{min}$ in less than two weeks. In type II, serum creatinine must be greater than $1.5 \mathrm{mg} / \mathrm{dl}$ or creatinine clearance $<40 \mathrm{ml} / \mathrm{min}$ but is more slowly progressive with a correspondingly better prognosis. Most patients pass through a sequence of ascites, followed by refractory ascites, and then hepatorenal syndrome. A large prospective study including patients with ascites demonstrated that hepatorenal syndrome developed in $18 \%$ at one year and in $39 \%$ at five years. ${ }^{2}$ Prognosis was poor with median survival of 1.7 weeks and $90 \%$ mortality at 10 weeks. The pathogenesis of hepatorenal syndrome is believed to involve splanchnic vascular dilatation with resultant vasoconstriction in other vascular beds particularly affecting the kidney. ${ }^{1}$ Therapies such as head out water immersion or liver transplantation can reverse this process but are not practical or rapidly available for many patients. $^{3}{ }^{4}$

Recent studies suggest that transjugular intrahepatic portosystemic stent-shunt (TIPS) or pharmacological therapy may be useful in this syndrome. The study by Brensing et al in this issue of $G u t^{5}$ describes the long term outcome in cirrhotics with hepatorenal syndrome treated with TIPS (see page 288). They divided their patients into those with hepatorenal syndrome types I and II. Three quarters responded to TIPS with improvement in renal function. One year survival in the treated group was $48 \%$. On an intention to treat basis, one year survival was 39\%. The survival of type II patients after TIPS was significantly better than that of type I patients (one year survival approximately $70 \%$ v $20 \%$ ). These results are very encouraging. However, it is important to remember that this was not a controlled trial. In addition, we are told little about the selection criteria for this cohort of patients. It is difficult to judge whether this group is directly comparable with the cohort identified prospectively by Gines et al, which most comprehensively defined the natural history of this syndrome. ${ }^{2}$ It is likely that the group with type I hepatorenal syndrome most closely resemble classical hepatorenal syndrome. Extrapolating from the graphs supplied, survival at 10 weeks in the type I hepatorenal syndrome group, by intention to treat, was 53\% compared with $10 \%$ described by Gines et al.

These results are very encouraging but controlled trials are required to confirm improvement in prognosis. The results of a small controlled trial of TIPS compared with large volume paracentesis in 25 patients with refractory ascites would cause some concern in this regard. It is probably directly relevant as refractory ascites is frequently a forerunner of hepatorenal syndrome. While ascites improved in many treated patients, overall survival was significantly lower in the TIPS group (29\% v 56\%) at two years. ${ }^{6}$ Four patients in each group had Pugh grade $\mathrm{C}$ liver disease. In the current study patients with serum bilirubin $>15 \mathrm{mg} / \mathrm{dl}$, a Pugh score $>12$, or spontaneous severe encephalopathy were excluded, which probably selected out some of the highest risk patients and may have improved the results.

Another choice for treating hepatorenal syndrome is long term vasoconstrictor therapy. A number of studies have shown that long term vasoconstrictor therapy improves renal function in patients with hepatorenal syndrome. These studies used the vasopressin analogues 
ornipressin or terlipressin (glypressin) or the alpha adrenoceptor agonist midodrine.$^{7-9}$ Intravenous $20 \%$ albumin was used to increase intravascular volume. The long acting somatostatin analogue octreotide was added in one study to inhibit production of endogenous vasodilator peptides. Renal function improved in the majority of patients treated and one study suggested improved survival compared with historical controls treated with low dose dopamine and volume repletion. ${ }^{7}$ Ischaemic complications led to treatment withdrawal in a small number of patients.

Hepatorenal syndrome is a manifestation of advanced decompensated liver disease. It may be unrealistic to expect TIPS or pharmacological therapy to improve long term prognosis if underlying liver disease is unaffected. Acute alcoholic hepatitis may be an exception where liver function could improve substantially with abstinence. The gold standard treatment for hepatorenal syndrome in patients with cirrhosis is likely to remain liver transplantation. Long waiting lists or contraindications such as active alcoholism may preclude this option in many patients, at least in the short term. TIPS or pharmacological therapy may act as a bridge to transplantation for these patients. There may also be additional benefits. Renal failure remains a major adverse prognostic factor for survival following liver transplantation. ${ }^{10}$ If renal failure per se is a critical determinant of prognosis following liver transplant, reversal of hepatorenal syndrome prior to transplantation could improve prognosis after transplant.

These are exciting times for the treatment of hepatorenal syndrome. Future clinical trials will need to confirm that these new therapies actually improve prognosis. Studies will also need to address how best to use these treatments and to determine whether they should be viewed as alternative or complementary therapies. It is also important to remember that hepatorenal syndrome frequently develops in hospital and may be precipitated by over diuresis, infection, or use of nephrotoxic drugs such as aminoglycosides, etc. Renal failure may be preventable in many of these patients. A recent trial has shown that intravenous albumin reduces the risk of renal failure and mortality in cirrhotic patients with spontaneous bacterial peritonitis. ${ }^{11}$ Hopefully we will see more advances in prevention and treatment of this syndrome.

Liver Unit, St Vincent's University Hospital,

P A MCCORMICK

Dublin 4, Ireland

1 Arroyo V, Gines P, Gerbes AL, et al. Definition and diagnostic criteria of refractory ascites and hepatorenal syndrome in cirrhosis. Hepatology refractory ascites

2 Gines A, Escorsell A, Gines P, et al. Incidence, predictive factors, and prognosis of the hepatorenal syndrome in cirrhosis with ascites. Gastroenterology 1993;105:229-36.

3 Yersin B, Burnier M, Magnenat P. Improvement of renal failure with repeated head-out water immersions in patients with hepatorenal syndrome associated with alcoholic hepatitis. Am $\mathcal{F}$ Nephrol 1995;15:260-5.

4 Jeyarajah DR, Gonwa TA, McBride M, et al. Hepatorenal syndrome: Combined liver kidney transplants versus isolated liver transplant. Transplantation 1997;64:1760-5.

5 Brensing KA, Textor J, Perz J, et al. Long term outcome after transjugular intrahepatic portosystemic stent-shunt in non-transplant cirrhotics with hepatorenal syndrome: a phase II study. Gut 2000; 47:288-95.

6 Lebrec D, Giuily N, Hadengue A, et al. Transjugular intrahepatic portosystemic shunts: comparison with paracentesis in patients with cirrhosis and temic shunts: comparison with paracentesis in patients with cirr
refractory ascites: a randomized trial. $\mathcal{F}$ Hepatol $1996 ; 25: 135-44$.

7 Angeli P, Volpin R, Gerunda G, et al. Reversal of type 1 hepatorenal syndrome with the administration of midodrine and octreotide. Hepatology syndrome with the
$1999 ; 29: 1690-7$.

8 Gulberg V, Bilzer M, Gerbes AL. Long-term therapy and retreatment of hepatorenal syndrome type 1 with ornipressin and dopamine. Hepatology 1999;30:870-5.

9 Hadengue A, Gadano A, Moreau R, et al. Beneficial effects of the 2-day administration of terlipressin in patients with cirrhosis and hepatorenal syndrome. F Hepatol 1998;29:565-70.

10 Lafayette RA, Pare G, Schmid CH, King AJ, Rohrer RJ, Nasaway SA. Pretransplant renal dysfunction predicts poorer outcome in liver transplantation. Clin Nephrol 1997;48:159-64.

11 Sort P, Navasa M, Aroyo V, et al. Effect of intravenous albumin on renal impairment and mortality in patients with cirrhosis and spontaneous bacterial peritonitis. N Engl f Med 1999;341:403-9. 